\title{
7 De Novo Multiple Dural Arteriovenous Report Fistulae Successfully Treated with Transarterial Embolization Using Low- concentration N-butyl-2-cyanoacrylate: A Case Report
}

Daisuke Izawa, Osamu Masuo, Takumi Kawaguchi, Rie Yako, and Naoyuki Nakao

Objective: We describe a case of de novo dural arteriovenous fistula (dAVF) developed after bilateral neck clipping for middle cerebral artery (MCA) aneurysms, treated with transarterial embolization (TAE) using liquid materials.

Case Presentation: A60-year-old man underwent neck clipping for a right ruptured MCA aneurysm, and a left unruptured MCA aneurysm. Follow-up MRA 1 year after the clipping showed an abnormal enlarged bilateral occipital artery (OA). Onyx was partially penetrated to parasinus, but low-concentration n-butyl-2-cyanoacrylate (NBCA) was widely penetrated to parasinus. After that, developed memory disturbance gradually worsened. The angiography demonstrated dAVF at transverse sinus, sigmoid sinus (SS), sinus confluence, and superior sagittal sinus with numerous cortical venous reflux. He was treated with TAE with liquid in two sessions. After embolization, cortical venous reflux completely disappeared and his cognitive dysfunction could be rapidly resolved. One year after the treatment, the angiogram revealed no recurrence of cortical venous reflux and he could maintain his independence.

Conclusion: In this case, bilateral craniotomy for neck clipping caused "de novo" dAVF presenting with cognitive dysfunction. In addition, TAE using NBCA was very useful to cure multiple dAVF.

Keywords $>$ de novo dural arteriovenous fistula, multiple shunts, transcatheter arterial embolization, low-concentration n-butyl-2-cyanoacrylate

\section{Introduction}

Dural arteriovenous fistula (dAVF) is an acquired disease caused by iatrogenic factors, traumatic factors, venous sinus thrombosis, and venous hypertension. ${ }^{1,2)}$ In most patients, the course is favorable. Some patients, however, show aggressive features with cortical venous reflux. In such patients, the annual incidence of intracranial hemorrhage is

Department of Neurosurgery, Wakayama Medical University, Wakayama, Wakayama, Japan

Received: January 1, 2018; Accepted: June 29, 2018

Corresponding author: Daisuke Izawa. Department of Neurosurgery, Wakayama Medical University, 811-1 Kimiidera, Wakayama, Wakayama 641-0012, Japan

Email:dizawa@wakayama-med.ac.jp

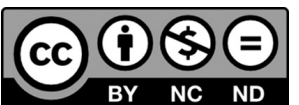

This work is licensed under a Creative Commons Attribution-NonCommercialNoDerivatives International License.

(C)2018 The Japanese Society for Neuroendovascular Therapy approximately $20 \%$, and that of non-hemorrhagic central neuropathy is approximately $11 \%{ }^{3}$ In this study, we report a patient with multiple de novo dAVF following bilateral craniotomy, with progressing dementia. We report the efficacy of transarterial liquid embolization in relation to published literature.

\section{Case Presentation}

A 60-year-old man had progressive memory disturbance. He had undergone neck clipping for a right ruptured middle cerebral artery (MCA) aneurysm 3 years previously, and left unruptured MCA aneurysm 2 years previously. In two sessions of craniotomy, the bilateral superficial temporal arteries (STA) were cut at their origins, but there were no sinus or cerebral venous injuries during surgery. In the perioperative period, no procedures that could induce restriction of venous return, such as catheter insertion into the jugular vein. 

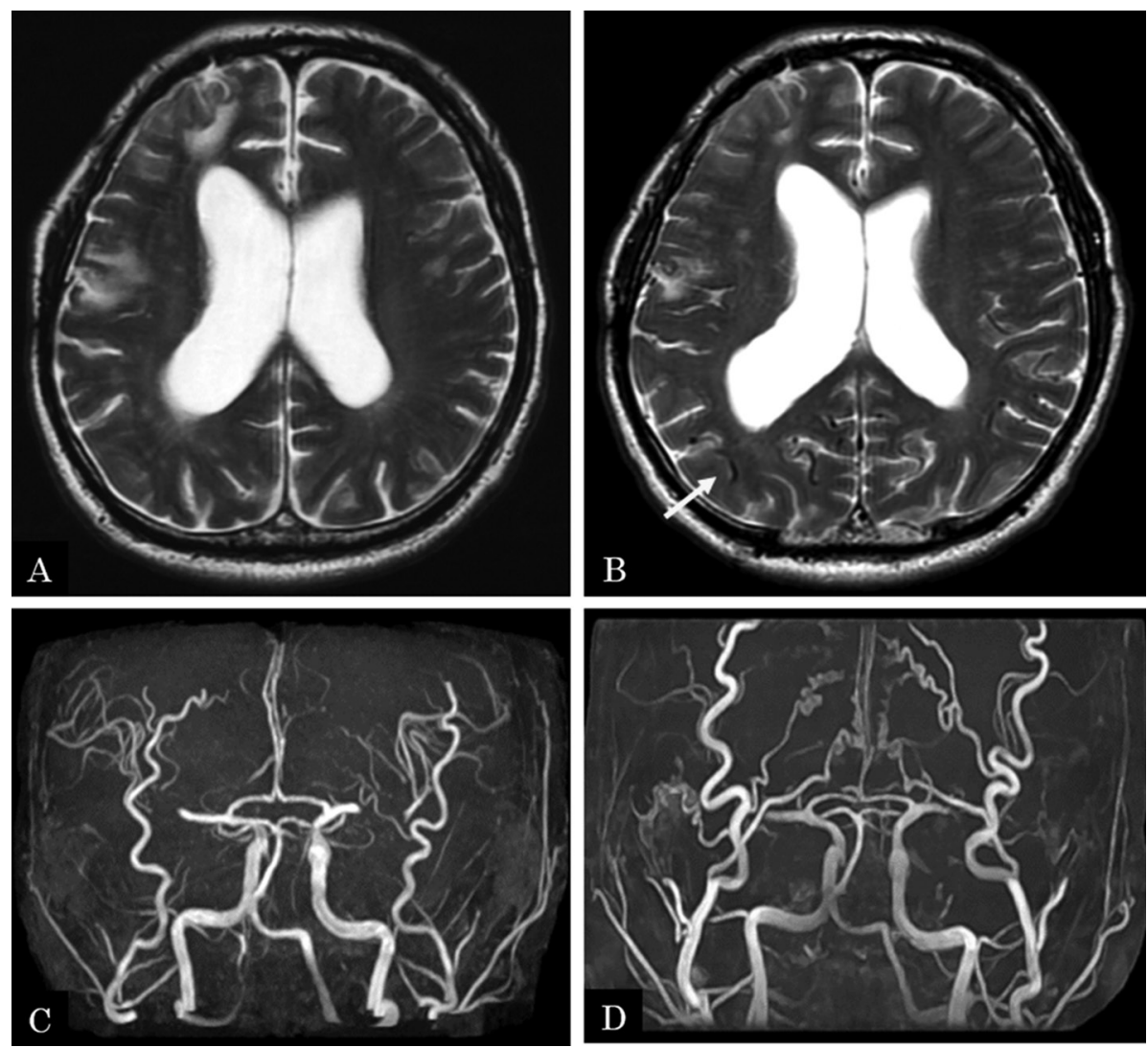

Fig. 1 MRI-T2-weighted image 3 months after clipping for left MCA aneurysm shows no anomalies and no dAVF (A), but 1 year later MRI-T2-weighted image (B) shows abnormal dilation of cerebral vessels representing venous congestion (arrow). Sequential change in MR angiography shows the tortuous growth of bilateral OA in 1 year (C and $\mathbf{D})$. dAVF: dural arteriovenous fistula; MCA: middle cerebral artery; OA: occipital artery

MRA revealed dilation of the bilateral occipital arteries (OA), which became markedly so (Fig. 1C and 1D) during the 2-year postoperative follow-up at the outpatient clinic. There was no new edematous change on any T2weighted MR images of the brain, but a large number of flow voids had appeared, suggesting dilation of superficial cerebral veins (Fig. 1A and 1B). Progressive memory disorder was observed, so cerebral angiography was performed for detailed examination.

On outpatient examination 14 days before admission, the Mini Mental State Examination (MMSE) score was $12 / 30$ points (items for the current place, name, reading, and language understanding were all correct). After admission, however, it was difficult to measure the MMSE score, and rapid progression of cognitive dysfunction was noted (fullerton advanced balance $[\mathrm{FAB}]=3 / 18$ points, Kohs block design test, and Raven's Colored Progressive Matrices Test were unmeasurable).

Cerebral angiography (external carotid artery [ECA] angiography) revealed multiple dAVF fed by the bilateral middle meningeal arteries (MMA) and OA and drained into the numerous dilated cortical veins via the parasinus due to the occlusion of superior sagittal sinus (SSS) (Fig. 2A and 2B). In addition, occlusion of the left transverse sinus (TS) and tight stenosis of the right TS were noted (Fig. 2C and 2D). Bilateral internal carotid angiography then showed marked cerebral venous congestion related to reflux to these cortical veins (Fig. 3). On the other hand, dAVF located at the ipsilateral sigmoid sinus (SS) was fed by the bilateral MMA and drained into the antegrade jugular vein. Based on these findings, the patient's cognitive dysfunction was closely related to SSS dAVF with cortical venous reflux (Fig. 4). However, these affected sinuses were not visualized as the original sinuses, and parasinus-mediated cortical reflux was observed. Considering that sufficient curative effects could not be achieved by transvenous embolization (TVE), transarterial embolization (TAE) with a liquid embolic material was selected. After proximal occlusion by transarterial liquid embolization for flow reduction, TAE with low-concentrated n-butyl2-cyanoacrylate (NBCA) was planned to completely cure SSS dAVF. 

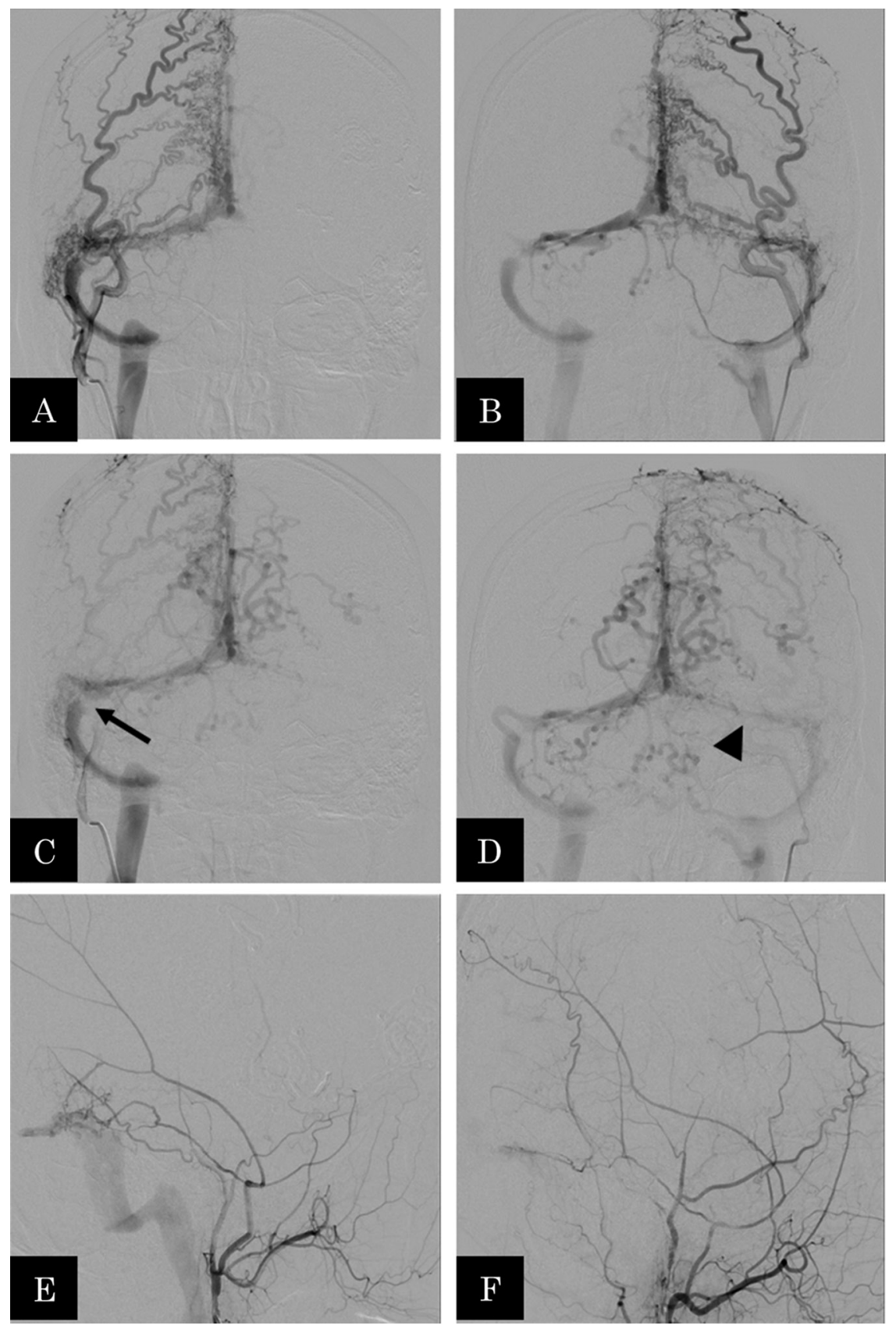

Fig. 2 Arterial phase of anteroposterior view angiography, right (A) and left OA (B), shows multiple shunt dAVF in wall of TS, SS, sinus confluence, superior sagittal sinus. Venous phase of anteroposterior view angiography, right (C) and left OA (D), shows right transverse sinus occlusion (arrow) and left transverse sinus stenosis, therefore accompany with retrograde venous drainage toward numerous superficial cortical veins of bilateral cerebral hemisphere and deep venous system through the straight sinus. Lateral view angiography of right middle meningeal artery $(E)$ and left middle meningeal artery $(F)$ shows fistulous communications in wall of TS-SS and antegrade venous drainage to each jugular vein. dAVF: dural arteriovenous fistula; OA: occipital artery; SS: sigmoid sinus; TS: transverse sinus

\section{First session of treatment}

Arteries supplying the SS, TS, and SSS (upper area) were targeted through the peripheries of the bilateral OA. Under general anesthesia, a 6 Fr. sheathless NV (Asahi Intecc, Co., Ltd., Aichi, Japan) was initially inserted into the right
ECA via the right femoral artery. Using a 4 Fr. Cerulean (Medikit, Tokyo, Japan) as an intermediate catheter, a microcatheter (Marathon, Medtronic, Minneapolis, MN, USA) was guided into the branch of right OA feeding SSS dAVF and TS dAVF. Embolization with Onyx (ev3, Irvine, CA, USA) 

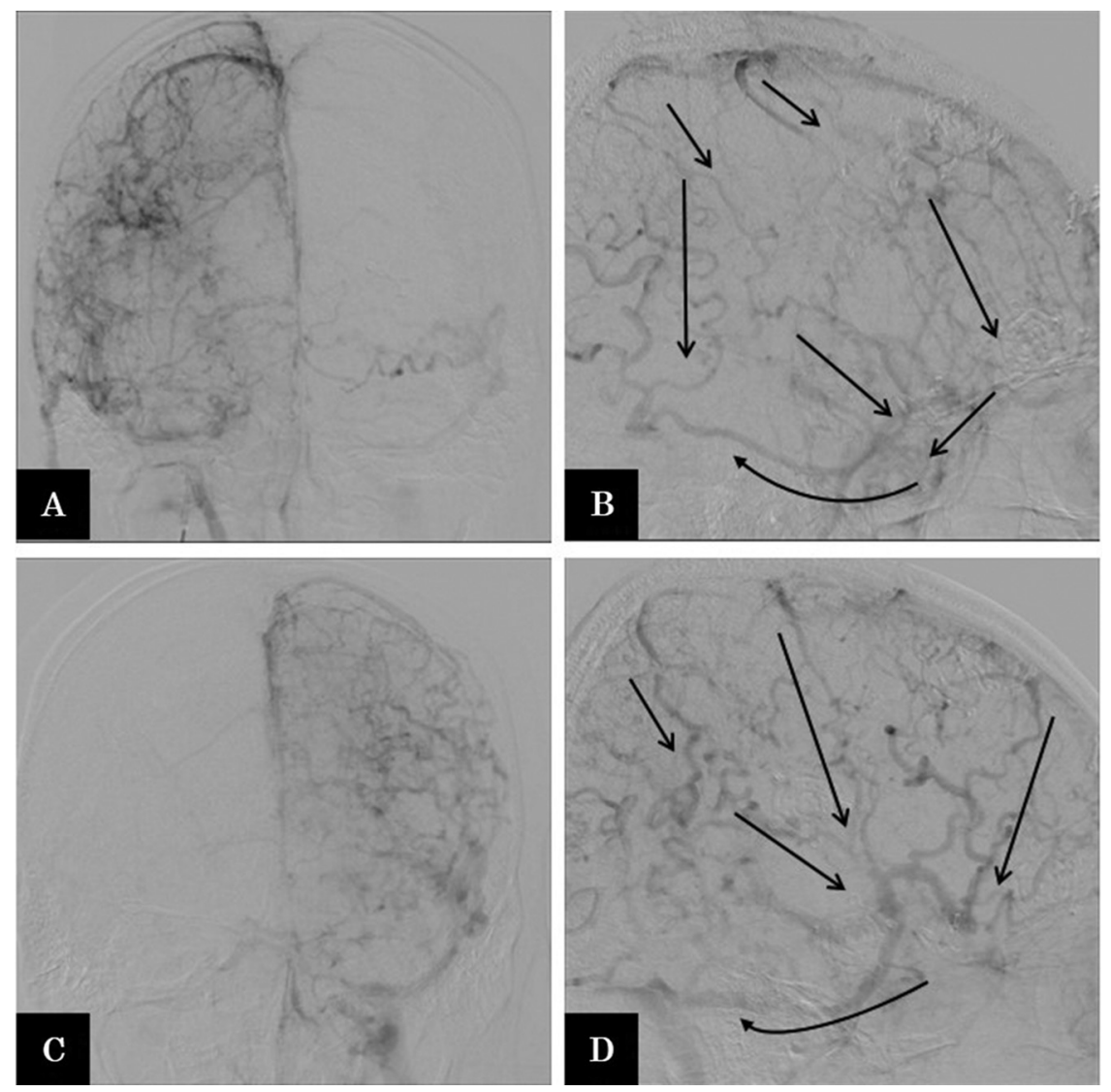

Fig. 3 Venous phase of right ICA angiography (A) anteroposterior view, (B) lateral view shows the retrograde venous drainage into numerous superficial cortical veins of right cerebral hemisphere. Left ICA angiography (C) anteroposterior view, (D) lateral view, same as right ICA angiography, shows the retrograde venous drainage of left cerebral hemisphere. ICA: internal carotid artery

was performed for shunt flow reduction. Subsequently, the same procedure of transarterial liquid embolization for flow reduction was performed for the several branches of the bilateral OA. After flow reduction was confirmed, the first session was considered to be complete.

\section{Second session of treatment}

The OA branch, extensively drained into the sinus confluence and the lower area of the SSS, was selected as the target vessel. Lower concentration of NBCA (13\%) was used in this session to completely occlude SSS dAVF.

A 7 Fr. Envoy catheter was inserted into the right OA through the right femoral artery under general anesthesia. With assistance by $4 \mathrm{Fr}$. Cerulean, a microcatheter (marathon) was inserted into the branch of OA, which is selected as the target vessel, more to the distal. Then, 13\% heated NBCA was infused slowly and intermittently. The NBCA solution was penetrated into not only the ipsilateral other feeding arteries, but also the contralateral feeding arteries in a retrograde fashion via the parasinus. Infusion time under DSA was 11 minutes. Embolization was completed, with a total $13 \%$ NBCA-Lipiodol solution volume of $1.7 \mathrm{~mL}$. Angiography immediately after embolization confirmed the disappearance of shunts from the right OA. Embolization for two branches of the left OA, in which shunt flow still remained, was also conducted as using the same procedure. Finally, cortical venous reflux from a large number of shunts involving the SSS, straight sinus, and sinus confluence, completely disappeared although an antegrade shunt at the SS remained (Fig. 5).

After curative liquid embolization, there were no new neurologic deficits. Transient alopecia was noted, but severe dermal complications, such as discoloration of the scalp, ulceration, or necrosis were not evident. A higher brain function test was conducted 10 days after surgery. MMSE and FAB scores were $21 / 30$ and $7 / 18$ points, respectively. Kohs block design test showed an intelligence quotient (IQ) of 45, and Raven's Colored Progressive Matrices Test 


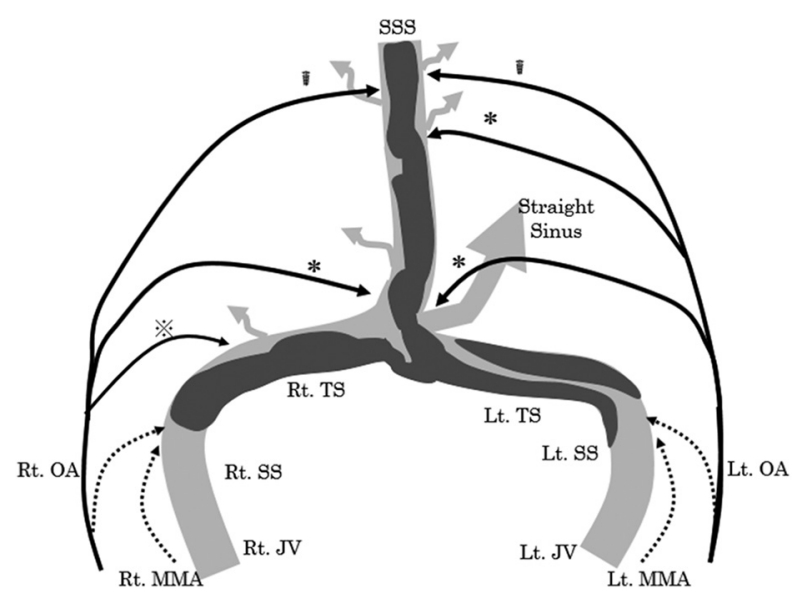

Fig. 4 Schema of neuroradiologic finding on anteroposterior projection. Obstruction of Sinus confluence, SSS, and right transverse sinus, and stenosis of left transverse sinus was revealed. Bilateral OA supplies fistulous communications in parasinus of TS-SS, sinus confluence, SSS, and retrograde venous drainage including numerous superficial cortical veins of bilateral cerebral hemisphere and deep venous system through the straight sinus. Meanwhile, bilateral proximal OA branch and MMA supplies fistulous communications in wall of SS and antegrade venous drainage to each JV. ※: Feeder of the first embolization used Onyx. ii: Feeder of the first session embolization used $17 \%$ NBCA. *: Feeder of the second session embolization used 13\% NBCA. NBCA: n-butyl-2-cyanoacrylate; JV: jugular vein; MMA: middle meningeal artery; OA: occipital artery; SSS: superior sagittal sinus; TS: transverse sinus; SS: sigmoid sinus

indicated a score of 27/36 points; a marked improvement in cognitive dysfunction was achieved. The patient was discharged seven days after embolization. After 1 year, cerebral angiography confirmed that disappearance of the shunt at SSS was maintained (Fig. 6). In addition, the modified Rankin Scale (mRS) score, before admission was 4, and it gradually improved to 1 after discharge.

\section{Discussion}

Acquired factors, such as venous hypertension and sinus thrombosis, may be involved in the pathogenesis of dAVF.,5) Post-craniotomy dAVF at the site of craniotomy also may be associated with an increase in the venous pressure, such as surgical-operation-related sinus compression/stenosis or sinus thrombosis. In the present case, however, dAVF developed at sites differing from the site of craniotomy. A previous study reported that distal venous hypertension or changes in the intracranial pressure were involved in the manifestation of physiological arteriovenous shunts, $\left.{ }^{6}\right)$ but the pathogenesis remains to be clarified. On the other hand, according to several studies, angiogenic factors, such as vascular endothelial growth factor (VEGF) and basic fibroblast growth factor (bFGF) can be related to the development of dAVF. The process of tissue repair/neovascularization after sinus thrombosis, focal inflammation of arteries/veins penetrating the skull, and an increase in blood flow in the ECA are reportedly involved in the enhancement of VEGF and bFGF expressions. ${ }^{4,7}$ In the present case, MRA showed a serial increase in OA signals. Multiple dAVF at the sinus, which was not involved in craniotomy, therefore appeared. In this case, there were no perioperative complication that may influence the cerebral veins or sinus. A load on the bilateral OA in the process of scalp wound healing related to bilateral STA cutting may therefore have led to multiple extensive dAVF through neovascularization factors, as described above.

Multiple dAVF, being relatively rare, account for approximately $7 \%$ of all dAVF. However, the risks of intracranial hemorrhage and non-hemorrhagic central neuropathy are higher than in the presence of a solitary dAVF, and cortical venous reflux is observed in many patients; therefore, treatment is performed in many patients. ${ }^{8)}$ The pathophysiology of multiple dAFV is complex, so a radical cure is difficult to achieve by TVE alone. In such patients, various therapeutic strategies, including TVE under craniotomy, several sessions of TAE targeting flow reduction, and gamma knife treatment, should be considered.

The treatment must be considered in the present case because progressive cognitive symptoms were observed. Concerning the pathogenesis, sinus packing may have resulted in aggravation of venous congestion on the contrary due to residual parasinus-mediated venous reflux. As it can be difficult to achieve radical cure by TVE, radical TAE with a liquid embolic material was planned. Recently, the Onyx, a liquid embolic material, for embolization of dAVF was reported as being useful. However, it is not covered by the Japanese health insurance. Lowconcentration heated NBCA was therefore as the embolic material. There are further advantages of low-concentration heated NBCA. If the target vessel is small and/or tortuous, low-concentration heated NBCA is thought to be more controllable in reaching the lesion site than Onyx. Second, the hardening mechanism of NBCA involves a polymerization reaction with inflammation. Even if TAE results in incomplete occlusion, the healing related to delayed thrombosis may cause complete occlusion. $\left.{ }^{9}\right)$ Liquid embolization in the present case was by the "plug and push method," which is usually used in embolization with Onyx. This method (using NBCA instead) is possible because low concentration can reduce the adhesiveness, 

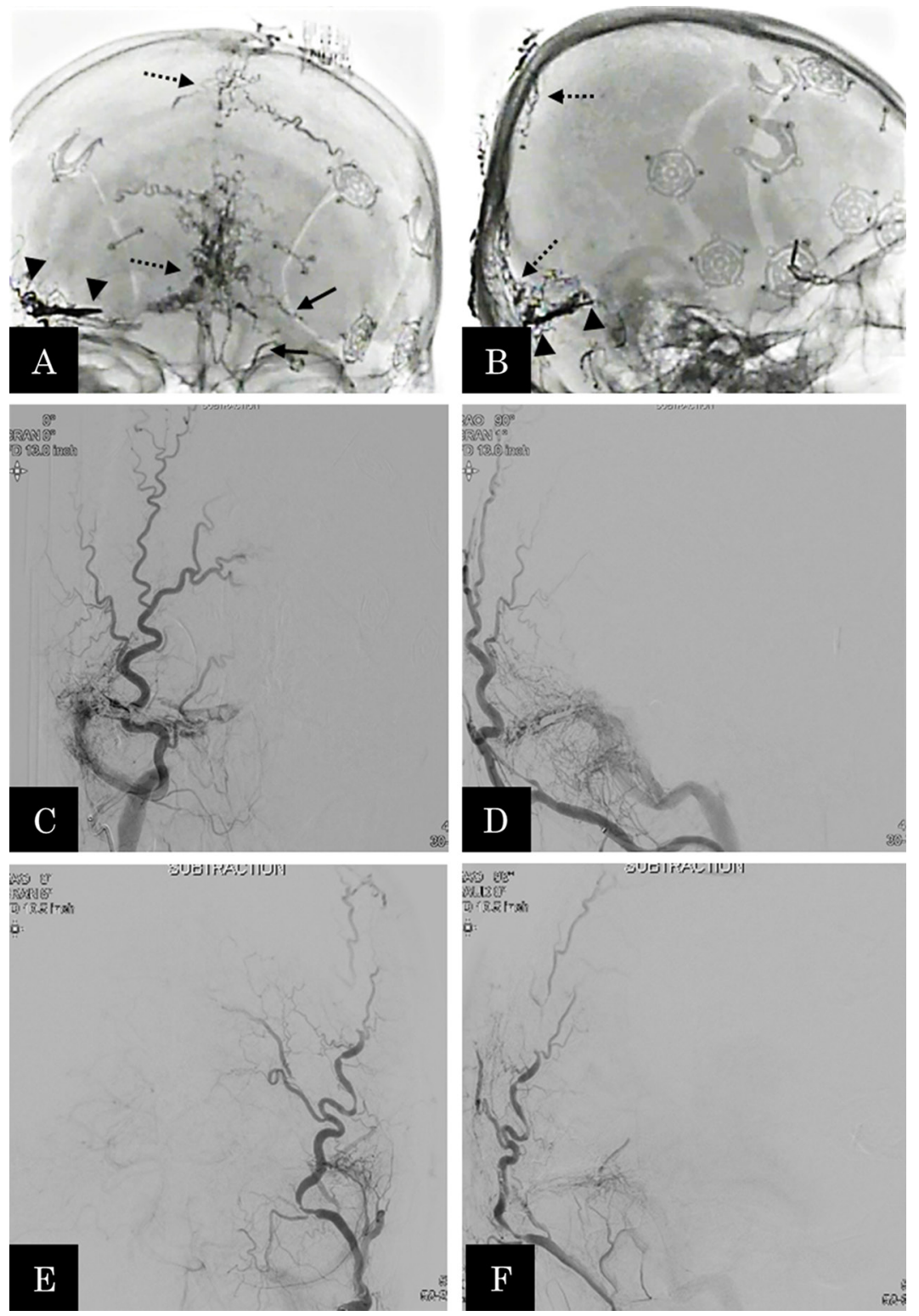

Fig. 5 Angiographic single shot image (A) anteroposterior view, (B) lateral view, Onyx cast was only penetrated to a part of parasinus (arrowheads), but $13 \%$ n-butyl2-cyanoacrylate cast was widely penetrated to the parasinus (dashed arrows) and contralateral occipital artery branches (solid arrow). Post-therapeutic DSA of right occipital artery, anteroposterior view (C) and lateral view (D), shows disappearance of cortical venous reflux. As with the left occipital artery DSA, anteroposterior view $(\mathrm{E})$ and lateral view $(\mathbf{F})$.

and heat can reduce the viscosity of Lipiodol. As a result, $13 \%$ NBCA with heating is thought to be useful from the viewpoints of adhesiveness and visibility. In addition, it is important to navigate the microcatheter into the more distal portion under intermediate-catheter assistance to minimize NBCA reflux. However, this procedure usually requires high radiation exposure. In the present case, the procedure was performed under DSA (3 frames/second) at a low radiation dose to reduce exposure, utilizing the characteristics of low-concentration NBCA: a high lipiodol content and relatively favorable visibility. Total exposure dose could be reduced to 5 Gy or below, and radiation-related complications could be minimized.

As a result, low-concentration NBCA was penetrated into not only ipsilateral and contralateral feeders but also parasinus as the site of shunts. This led to the disappearance of cortical venous reflux and an improvement in cognitive symptoms. 

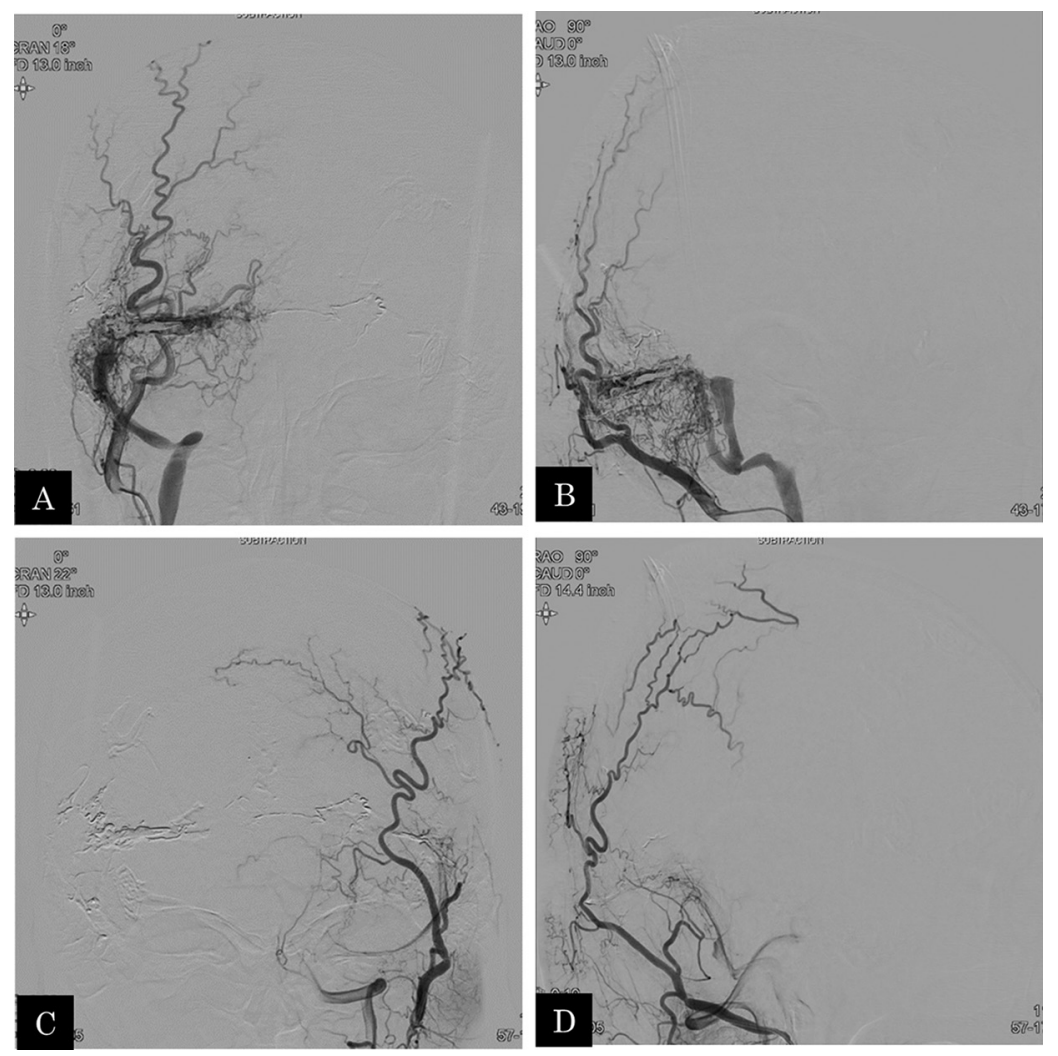

Fig. 6 Follow-up digital subtractive angiography obtained 1 year after the latest treatment shows no recurrence of cortical venous reflux. Right occipital artery angiography, anteroposterior view (A) and lateral view (B), left occipital artery angiography, anteroposterior view (C) and lateral view (D).

\section{Conclusion}

TAE with low-concentration heated NBCA for multiple dAVF led to the disappearance of cortical venous reflux in our patient. For TAE with a liquid embolic material, it is important to understand the characteristics of each liquid embolic material.

\section{Disclosure Statement}

There are no conflicts of interest regarding this article.

\section{References}

1) Awad IA, Little JR, Akarawi WP, et al: Intracranial dural arteriovenous malformations: factors predisposing to an aggressive neurological course. J Neurosurg 1990; 72: 839-850.

2) Lasjaunias $P$, Chiu $M$, ter Brugge $K$, et al: Neurological manifestations of intracranial dural arteriovenous malformations. J Neurosurg 1986; 64: 724-730.
3) Gupta A, Periakaruppan A: Intracranial dural arteriovenous fistulas: a review. Indian J Radiol Imaging 2009; 19: 43-48.

4) Li Q, Zhang Q, Huang QH, et al: A pivotal role of the vascular endothelial growth factor signaling pathway in the formation of venous hypertension-induced dural arteriovenous fistulas. Mol Med Rep 2014; 9: 1551-1558.

5) Terada T, Higashida RT, Halbach VV, et al: Development of acquired arteriovenous fistulas in rats due to venous hypertension. J Neurosurg 1994; 80: 884-889.

6) Nishimoto Y, Takahashi K, Hayashi S, et al: [Two cases of dural arteriovenous fistula that developed after surgery in sited remote from the craniotomy]. Jpn J Neurosurg 2014; 23: 667-671. (in Japanese)

7) Uranishi R, Nakase H, Sakaki T: Expression of angiogenic growth factors in dural arteriovenous fistula. J Neurosurg 1999; 91: 781-786.

8) van Dijk JM, TerBrugge KG, Willinsky RA, et al: Multiplicity of dural arteriovenous fistulas. J Neurosurg 2002; 96: 76-78.

9) Sugiu K, Hiramatsu M, Tokunaga K, et al: [Intra-cranial dural arteriovenous fistula: classification and treatment]. Jpn J Neurosurg 2013; 22: 37-43. (in Japanese) 